\title{
Estudio de la descomposición térmica de compuestos tipo hidrotalcita
}

\author{
I.NEBOT-DÍAZ", V.RIVES", J.ROCHA"*;, J.B.CARDA* \\ * Dpto. Química Inorgánica y Orgánica, Universitat Jaume I, Castellón \\ ** Dpto. Química Inorgánica, Universidad de Salamanca, Salamanca \\ ${ }^{* * *}$ Dpto. Química, Universidade de Aveiro, Aveiro (Portugal)
}

Se ha estudiado la descomposición térmica de compuestos tipo hidrotalcita de fórmula $\mathrm{Mg}_{1-x} \mathrm{Al}_{\mathrm{x}}\left(\mathrm{CO}_{3}\right)_{x / 2} \cdot \mathrm{nH}_{2} \mathrm{O}$ en función de la relación molar $(\mathrm{x})$ entre los cationes $\mathrm{Mg}$ y Al. Los compuestos sintetizados mediante la vía de coprecipitación a pH constante, se han calcinado a diferentes temperaturas comprendidas entre 500 y $1200^{\circ} \mathrm{C}$ con diferentes tiempos de retención. Este estudio se ha realizado mediante las técnicas de Análisis Térmico Diferencial y Termogravimétrico, Difracción de Rayos X, Microscopía Electrónica de Barrido y Resonancia Magnética Nuclear de ${ }^{27} \mathrm{Al}$.

Los principales resultados muestran que la relación molar óptima (x) para la formación de la fase hidrotalcita está comprendida entre los valores de 0.20 y 0.35 , generándose como productos de descomposición la espinela $\left(\mathrm{MgAl}_{2} \mathrm{O}_{4}\right)$ y la periclasa $(\mathrm{MgO})$.

Palabras clave: hidrotalcita, espinela, descomposición térmica.

\section{Thermal decomposition study of hydrotalcite-like compounds}

Thermal decomposition of hydrotalcite-like compounds with formula $\mathrm{Mg}_{\mathrm{x}} \mathrm{Al}_{1-\mathrm{x}}\left(\mathrm{CO}_{3}\right)_{\mathrm{x} / 2} \cdot \mathrm{nH}_{2} \mathrm{O}$ has been studied varying the molar ratio $(\mathrm{x})$ between $\mathrm{Mg}$ and $\mathrm{Al}$ cations. Compounds synthesized by coprecipitation method at fixed $\mathrm{pH}$, have been fired at different temperatures between 500 and $1200{ }^{\circ} \mathrm{C}$ with different remaining times. This study has been carried out by Differential Thermal and Thermogravimetric Analysis (DTA/TG), X-Ray Diffraction (XRD), Scanning Electron Microscopy (SEM) and ${ }^{27} \mathrm{Al}$ Nuclear Magnetic Resonance $\left({ }^{27} \mathrm{Al}-\mathrm{MAS}-\mathrm{NMR}\right)$

Main results show that the optimum molar ratio (x) to hydrotalcite phase formation is between 0.20 and 0.35 , obtaining spinel $\left(\mathrm{MgAl}_{2} \mathrm{O}_{4}\right)$ and Periclase $(\mathrm{MgO})$ as the decomposition compounds.

Key Words: Hydrotalcite, spinel, thermal decomposition.

\section{INTRODUCCIÓN}

Los compuestos tipo hidrotalcita, también conocidos como arcillas aniónicas, son hidróxidos dobles laminares con la fórmula general:

$\left[\mathrm{M}_{1-\mathrm{x}} \mathrm{M}^{\prime}(\mathrm{OH})_{2}\right]^{\mathrm{x}+} \mathrm{X}_{\mathrm{x} / \mathrm{n}}^{\mathrm{n}-} \cdot \mathrm{mH}_{2} \mathrm{O}$

donde $\mathrm{M}$ son cationes divalentes (tipo $\mathrm{Mg}, \mathrm{Zn}, \mathrm{Ni}, \mathrm{Co}, . .$. ), $\mathrm{M}^{\prime}$ son cationes trivalentes $(\mathrm{Al}, \mathrm{Fe}, \mathrm{Co}, \ldots)$ y $\mathrm{X}$ representa el anión de la intercapa $\left(\mathrm{CO}_{3}^{2-}, \mathrm{SO}_{4}^{2-}, \mathrm{NO}_{3}^{-}, \ldots\right)$.

La estructura de la hidrotalcita es similar a la estructura de la brucita, $\mathrm{Mg}(\mathrm{OH})_{2}$, donde octaedros de $\mathrm{Mg}^{2+}$ se unen por las aristas para formar capas. Estas capas se empaquetan unas encima de otras enlazadas por puentes de hidrógeno.

Cuando los iones $\mathrm{Mg}^{2+}$ son sustituidos por un ion trivalente de radio semejante ( $\mathrm{Al}^{3+}$ en el caso de la hidrotalcita) se genera una carga positiva en la capa de hidróxidos. Esta carga positiva neta se compensa por el anión $\mathrm{CO}_{3}{ }^{2-}$ que se sitúa en la región situada entre las dos capas de brucita. En el espacio libre de esta intercapa se localiza el agua de cristalización (figura 1).

Los compuestos tipo hidrotalcita presentan capacidad para el intercambio iónico, así como propiedades adsorbentes y catalíticas, lo que ha conducido a un gran interés por este tipo de productos como precursores de catalizadores. En este tipo de materiales, los cationes están muy próximos entre ellos, por lo que generalmente el proceso de calcinación per- mite obtener mezclas homogéneas de óxidos dispersos con elevada superficie específica [1-2].

La descomposición térmica de los compuestos tipo hidrotalcita se puede dividir en diferentes etapas, teniendo en cuenta que dichos productos son estables hasta una temperatura próxima a $\operatorname{los} 400^{\circ} \mathrm{C}$ [3]. A temperaturas inferiores a $200^{\circ} \mathrm{C}$, los compuestos tipo hidrotalcita pierden parte del agua localizada en la intercapa. A temperaturas comprendidas entre 200 y $450^{\circ} \mathrm{C}$ se desprenden los iones carbonatos y el sólido resultante está parcialmente deshidroxilado. Se forma la fase $\mathrm{Mg}_{6} \mathrm{Al}_{2} \mathrm{O}_{8}(\mathrm{OH})_{2}$. Si se calcina a temperaturas comprendidas entre 450 y $900^{\circ} \mathrm{C}$ esta fase se transforma en periclasa $(\mathrm{MgO})$ y espinela $\left(\mathrm{MgAl}_{2} \mathrm{O}_{4}\right)$ [4-10]. Sin embargo, si la calcinación es a $500^{\circ} \mathrm{C}$, se forma la fase $\mathrm{Mg}_{6} \mathrm{Al}_{2}(\mathrm{OH})_{2}$, que en presencia de agua y una fuente de carbonatos puede reconstruir el compuesto tipo hidrotalcita original [11].

Las reacciones que tienen lugar durante el tratamiento térmico de estos compuestos tipo hidrotalcita, son las siguientes:

$$
\begin{aligned}
& \mathrm{Mg}_{6} \mathrm{Al}_{2}(\mathrm{OH})_{16} \mathrm{CO}_{3} \cdot 4 \mathrm{H}_{2} \mathrm{O} \stackrel{25-250^{\circ} \mathrm{C}}{\longrightarrow} \mathrm{Mg}_{6} \mathrm{Al}_{2}(\mathrm{OH})_{16} \mathrm{CO}_{3}+4 \mathrm{H}_{2} \mathrm{O} \uparrow \\
& \mathrm{Mg}_{6} \mathrm{Al}_{2}(\mathrm{OH})_{16} \mathrm{CO}_{3} \stackrel{400-450^{\circ} \mathrm{C}}{\longrightarrow} \mathrm{Mg}_{6} \mathrm{Al}_{2} \mathrm{O}_{8}(\mathrm{OH})_{2}+7 \mathrm{H}_{2} \mathrm{O} \uparrow+\mathrm{CO}_{2} \uparrow \\
& \mathrm{Mg}_{6} \mathrm{Al}_{2} \mathrm{O}_{8}(\mathrm{OH})_{2} \stackrel{450-900^{\circ} \mathrm{C}}{\longrightarrow} \mathrm{MgAl}_{2} \mathrm{O}_{4}+5 \mathrm{MgO}+\mathrm{H}_{2} \mathrm{O} \uparrow
\end{aligned}
$$




\section{PROCEDIMIENTO EXPERIMENTAL}

\subsection{Preparación de muestras}

Se han preparado cuatro muestras con diferente relación $\mathrm{Mg} / \mathrm{Al}$ (tabla I). Los reactivos que se han utilizado son: $\mathrm{AlCl}_{3} \cdot 6 \mathrm{H}_{2} \mathrm{O}$ (Merck 99\%), $\mathrm{MgCl}_{2} \cdot 6 \mathrm{H}_{2} \mathrm{O}$ (Merck 99.5\%), $\left(\mathrm{NH}_{4}\right)_{2} \mathrm{CO}_{3}$ (Panreac). Todas las muestras estudiadas se han sintetizado empleando la metodología de coprecipitación a pH constante [9], empleando como fuente de carbonatos el $\left(\mathrm{NH}_{4}\right)_{2} \mathrm{CO}_{3}$ y como agente basificante el $\mathrm{NH}_{4} \mathrm{OH}$. Las disoluciones de partida han sido $\mathrm{MgCl}_{2} 0.28 \mathrm{M}, \mathrm{AlCl}_{3} 0.07 \mathrm{M}$ y $\left(\mathrm{NH}_{4}\right)_{2} \mathrm{CO}_{3}$ 0.25M. La disolución que contiene el $\mathrm{AlCl}_{3}$ y $\mathrm{MgCl}_{2}$ se ha adicionado lentamente sobre la disolución de $\left(\mathrm{NH}_{4}\right)_{2} \mathrm{CO}_{3}$, manteniendo constante el $\mathrm{pH}$ a 10 con la adición de $\mathrm{NH}_{4} \mathrm{OH}$. El precipitado obtenido se filtra y se seca a $70^{\circ} \mathrm{C}$, y posteriormente se calcina a una velocidad de $10^{\circ} \mathrm{C} \mathrm{min}^{-1}$ hasta $300,500,700,900$ y $1200^{\circ} \mathrm{C}$.

\subsection{Caracterización del material obtenido}

El análisis elemental del magnesio y aluminio se llevó a cabo mediante absorción atómica con un equipo Varian espectrAA 610.

Las muestras sintetizadas se han sometido a análisis térmico diferencial y termogravimétrico simultáneo (DTA/TG) en un equipo METTLER TOLEDO 851e, acoplado a un espectrómetro de masas BALZERS, para la determinación de los gases desprendidos durante el tratamiento térmico. Las medidas se han realizado en flujo de aire $\left(50 \mathrm{ml} \mathrm{min}^{-1}\right)$ con una velocidad de calentamiento de $10^{\circ} \mathrm{C} \mathrm{min}^{-1}$.

Las difracciones de Rayos $X$ se han realizado en un equipo SIEMENS D5000D en el intervalo $2 \theta 5-70^{\circ}$, y la identificación de fases se ha efectuado por comparación con las fichas JCDPS.

Se ha empleado la técnica de microscopía electrónica de barrido (MEB) para la caracterización microestructural de las muestras, utilizando un microscopio LEICA LEO440i acoplado con un espectrómetro de dispersión de energías de rayos $\mathrm{X}$ OXFORD. Estos equipos, anteriormente mencionados, pertenecen al Servei Central d'Instrumentació Científica de la Universitat Jaume I de Castellón.

La obtención de los espectros de resonancia magnética nuclear de ${ }^{27} \mathrm{Al}$ se han realizado con un equipo Bruker MSL 400P del Departamento de Química de la Universidad de Aveiro. Los espectros se han recogido a $104.3 \mathrm{MHz}$, con pulsos de radiofrecuencia de $0.6 \mu$ s (equivalente a $10^{\circ}$ ), tiempos de relajación de $1 \mathrm{~s}$, y velocidades de giro de $30 \mathrm{kHz}$.

\section{RESULTADOS Y DISCUSIÓN}

\subsection{Estudio del efecto de la relación $\mathrm{Mg}$ : $\mathrm{Al}$ en la composición de los compuestos tipo hidrotalcita.}

En los casos en los que la relación entre cationes es 4:1 ó 2:1, se observa mediante difracción de rayos $\mathrm{X}$ la formación de la fase hidrotalcita como única fase cristalina (figura 2). Cuando la relación $\mathrm{Mg}$ :Al es la estequiométrica de la espinela, es decir 1:2, la formación de la fase hidrotalcita disminuye drásticamente, pasando a ser una fase minoritaria en favor del $\mathrm{Al}(\mathrm{OH})_{3^{\prime}}$ bayerita. Esto es debido a que la fase hidrotalcita puede asumir una cantidad máxima de aluminio: mientras el octaedro $\mathrm{Mg}(\mathrm{OH})_{6}$ es eléctricamente neutro, el octaedro

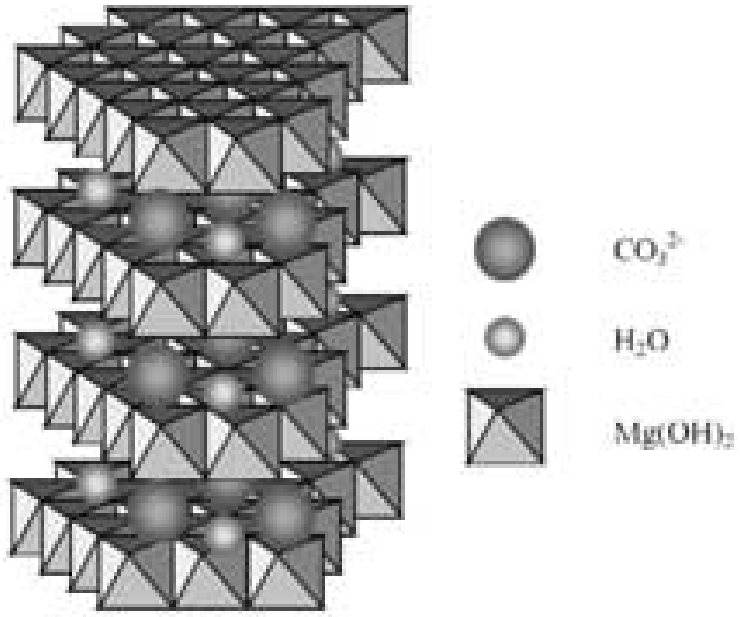

Figura 1. Estructura de la hidrotalcita

TABLA I. COMPOSICIÓN INICIAL DE LAS MUESTRAS

\begin{tabular}{|c|c|c|c|}
\hline Muestra & $\begin{array}{c}\text { Relación Mg:Al } \\
\text { Teórica }\end{array}$ & $\begin{array}{c}\text { Relación Mg:Al } \\
\text { Experimental }\end{array}$ & Fórmula nominal \\
Mg41 & $3.17: 1$ & $3.65: 1$ & $\mathrm{M}_{0.76} \mathrm{Al}_{0.24}\left(\mathrm{CO}_{3}\right)_{0.12}(\mathrm{OH})_{2} \cdot \mathrm{nH}_{2} \mathrm{O}$ \\
$\mathrm{Mg} 21$ & $1.87: 1$ & $2.11: 1$ & $\mathrm{M}_{0.65} \mathrm{Al}_{0.35}\left(\mathrm{CO}_{3}\right)_{0.17}(\mathrm{OH})_{2} \cdot \mathrm{nH}_{2} \mathrm{O}$ \\
$\mathrm{Mg} 12$ & $1: 1.87$ & $1: 2.25$ & $\mathrm{M}_{0.35} \mathrm{Al}_{0.65}\left(\mathrm{CO}_{3}\right)_{0.33}(\mathrm{OH})_{2} \cdot \mathrm{nH}_{2} \mathrm{O}$ \\
Mg14 & $1: 3.17$ & $1: 2.92$ & $\mathrm{M}_{0.24} \mathrm{Al}_{0.76}\left(\mathrm{CO}_{3}\right)_{0.38}(\mathrm{OH})_{2} \cdot \mathrm{nH}_{2} \mathrm{O}$ \\
\hline
\end{tabular}

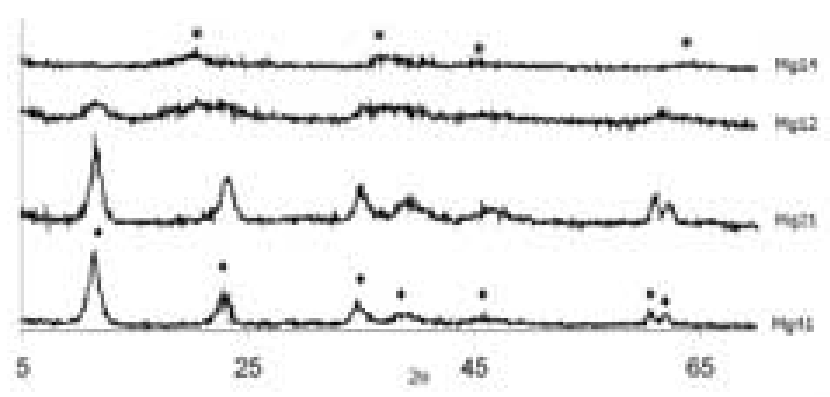

Figura 2. Diagramas DRX de las muestras en crudo $(\bullet=$ Hidrotalcita, *=Bayerita)

$\mathrm{Al}(\mathrm{OH})_{6}$ está cargado positivamente y la existencia de octaedros $\mathrm{Al}(\mathrm{OH})_{6}$ compartiendo aristas resulta inestable; por tanto todo el aluminio que no puede incorporarse a la estructura de la hidrotalcita queda en el medio de reacción, formándose en este caso el $\mathrm{Al}(\mathrm{OH})_{3}$ debido al $\mathrm{pH}$ básico necesario para la precipitación del $\mathrm{Mg}(\mathrm{OH})_{2}$ (figura 2).

Una de las diferencias más notables entre los análisis por difracción de las muestras sintetizadas, radica en las reflexiones correspondientes a la fase hidrotalcita, ya que a medida que se aumenta el contenido en aluminio, también lo hace la anchura de dichas reflexiones. Esto puede ser debido a la peor cristalización de la fase (también disminuye la intensidad de los picos), ya que la relación estequiométrica de la hidrotalcita es de Mg:Al 3:1. A mayor contenido de aluminio, menos favorecida está la formación de la fase hidrotalcita.

Esta observación se corrobora en las muestras con una relación de $\mathrm{Mg}$ :Al de 1:4, ya que la única fase que se detecta mediante difracción de rayos $X$ es la correspondiente a la bayerita (figura 2). 


\subsection{Estudio de la evolución térmica de los compuestos tipo hidrotalcita.}

Se ha registrado el análisis térmico (DTA/TG) y la espectrometría de masas para cada una de las muestras sintetizadas. Los resultados de estos análisis demuestran que en el caso de los menores contenidos en aluminio la estructura hidrotalcita está mejor formada, ya que la pérdida de los aniones $\mathrm{CO}_{3}{ }^{2-}$ y el agua de la intercapa, se produce a mayor temperatura que en el caso de las muestras con mayores contenidos en aluminio. De hecho, para las muestras con relación $\mathrm{Mg}: \mathrm{Al}$ de 4:1 y 2:1, el grupo $\mathrm{CO}_{3}{ }^{2-}$ y el agua de la intercapa se pierden a temperaturas superiores de $400^{\circ} \mathrm{C}$, mientras que en los otros dos casos, la temperatura de descomposición desciende hasta los $300^{\circ} \mathrm{C}$ (figura 3).

En todos los casos, se observa mediante los análisis obtenidos con el espectrómetro de masas, la presencia de una pequeña cantidad de cloruro procedente de los reactivos utilizados, que se elimina entre $750^{\circ}$ y $900^{\circ} \mathrm{C}$, dependiendo de la muestra. La mayor temperatura de volatilización del cloruro se obtiene para los menores contenidos de aluminio, lo que vuelve a confirmar la mejor formación de la fase hidrotalcita. Este cloruro, por su menor tamaño, debe situarse en la intercapa de la hidrotalcita junto con los $\mathrm{CO}_{3}{ }^{2-}$ (el espaciado interlaminar de hidrotalcitas con $\mathrm{Cl}^{-}$es prácticamente coincidente con el de hidrotalcitas con $\left.\mathrm{CO}_{3}^{2-}[1]\right)$.

La figura 4 incluye los diagramas de difracción de Rayos$\mathrm{X}$ de las muestras calcinadas a $1200^{\circ} \mathrm{C}$ sin tiempo de retención a dicha temperatura. Para las muestras Mg41 y Mg21, con relaciones molares $(\mathrm{x})$ comprendidas entre los valores teóricos de estabilidad de los compuestos tipo hidrotalcita (0.20-0.35), se observan como los productos de descomposición son exclusivamente los correspondientes a los teóricos de la hidrotalcita, es decir, periclasa $(\mathrm{MgO})$ y espinela $\left(\mathrm{MgAl}_{2} \mathrm{O}_{4}\right)$. En el caso de la muestra Mg41, debido a su mayor contenido en magnesio, presenta un formación mayor de periclasa.

Las muestras Mg12 y Mg14 tienen unos valores de $\mathrm{x}$ superiores a los teóricos de las hidrotalcitas, por lo que el producto de descomposición no es el propio de esta estructura. La única fase capaz de cristalizar durante el proceso de calcinación es la espinela $\mathrm{MgAl}_{2} \mathrm{O}_{4}$. Se observa (figura 5) que la temperatura de formación de la espinela es relativamente baja (comienza a $700^{\circ} \mathrm{C}$ ), comparada con la temperatura de síntesis necesaria por la ruta cerámica tradicional [12].

El estudio mediante ${ }^{27} \mathrm{Al}$ MAS-NMR, permite analizar la evolución del entorno cristalino del $\mathrm{Al}$ durante el proceso de descomposición térmica de los compuestos tipo hidrotalcita. En la figura 6 se representa dicha evolución para la muestra Mg12.

En la muestra cruda, se observa que todo el $\mathrm{Al}$ presente en la muestra se encuentra ocupando posiciones octaédricas (señal a 9 ppm), tal y como era de esperar, puesto que el $\mathrm{Al}$ se encuentra sustituyendo parcialmente al $\mathrm{Mg}$ en la brucita, con un entorno octaédrico, y el resto de $\mathrm{Al}$ que no ha formado parte de la estructura tipo hidrotalcita, se encuentra formando bayerita $\left(\mathrm{Al}(\mathrm{OH})_{3}\right)$, donde el $\mathrm{Al}$ también ocupa posiciones octaédricas. A medida que la muestra se va sometiendo a temperaturas de calcinación superiores, se observa que el Al pasa a ocupar posiciones tetraédricas, pero manteniendo parte del Al todavía en posiciones octaédricas. Este hecho indica que la espinela formada no posee una estructura de espinela normal ( $\mathrm{Mg}$ en posiciones tetraédricas y $\mathrm{Al}$ en posiciones octaédricas), sino que presenta cierto grado de inversión. Cuando la muestra se calcina a $1200^{\circ} \mathrm{C}$, la relación entre el $\mathrm{Al}$ octaédrico
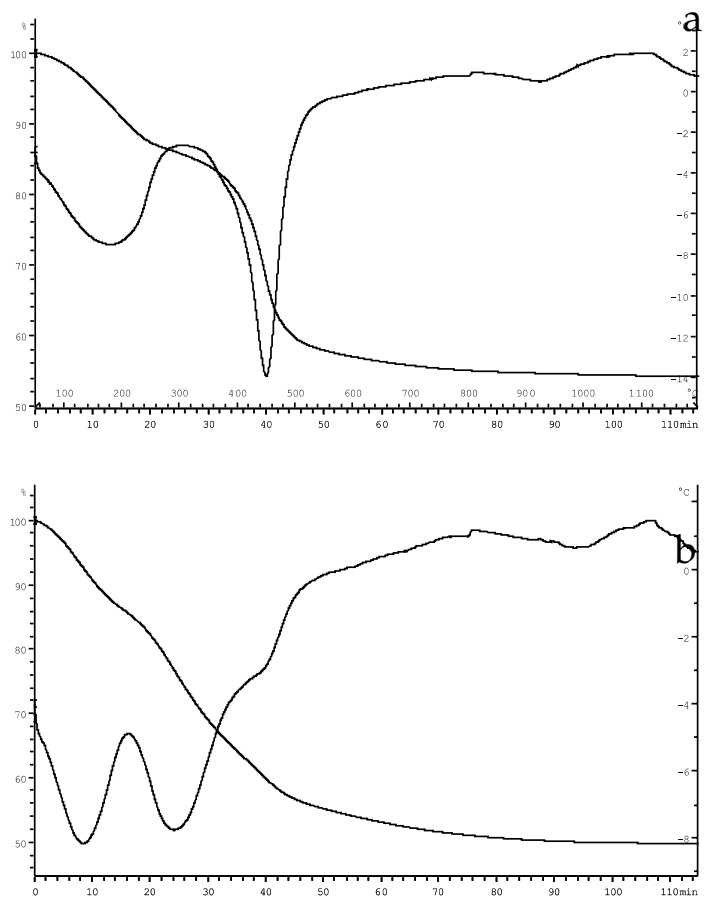

Figura 3. Análisis Térmico de la muestras a) Mg41; b)Mg14

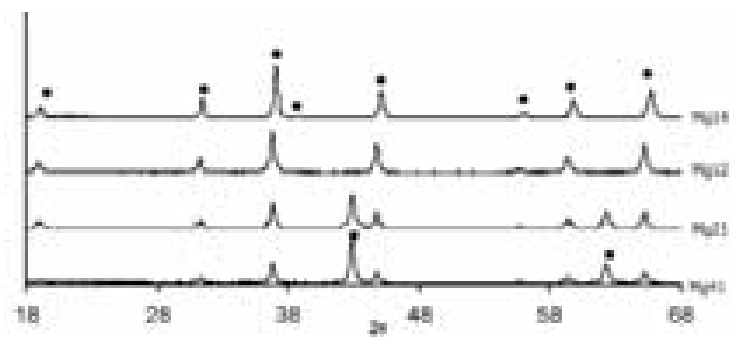

Figura 4. Diagramas DRX de las muestras calcinadas a $1200^{\circ} \mathrm{C}$ $(\bullet=$ Espinela, $\mathrm{n}=$ Periclasa)

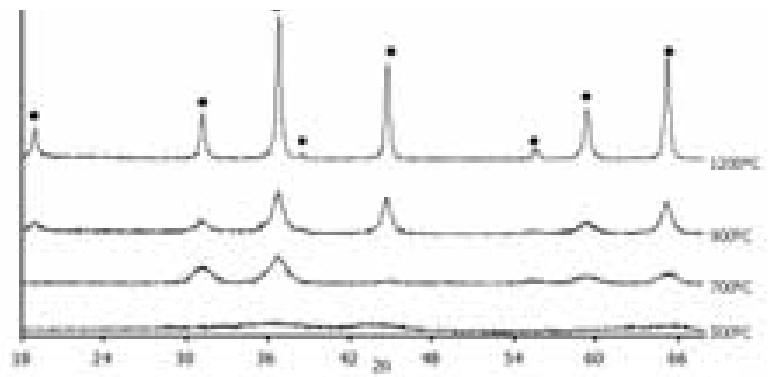

Figura 5.Diagramas DRX de la muestra Mg12 calcinada a diferentes temperaturas $(\bullet=$ Espinela $)$

y el Al tetraédrico aumenta de nuevo, lo que indica que a esta temperatura la espinela presenta un grado de inversión menor y tiene una estructura más próxima a la propia de la espinela normal, que es la que le corresponde (tabla II). A dicha temperatura se observa un desdoblamiento en la señal de $\mathrm{Al}$ octaédrico a 9.1 y 3.5 ppm; esto puede ser debido a la presencia de dos entornos octaédricos ligeramente diferentes para el Al. 
En la figura 7 se incluye una micrografía obtenida mediante microscopia electrónica de barrido, en la que se observa la cristalización cúbica de la espinela en el proceso de calcinación a $1200^{\circ} \mathrm{C}$ de la muestra Mg12.

\section{CONCLUSIONES}

El método de síntesis establecido permite obtener compuestos con estructura tipo hidrotacita para valores de relación molar entre el $\mathrm{Mg}$ y $\mathrm{Al}$ superior al valor teórico máximo de $x=0.35$, si bien se forman simultáneamente otras fases como la bayerita, debido al exceso de $\mathrm{Al}$ y al medio básico en el que se trabaja. La descomposición térmica de este tipo de productos genera periclasa y espinela en función de la relación molar entre $\mathrm{Mg}$ y $\mathrm{Al}$ de la que se parta. El estudio mediante ${ }^{27} \mathrm{Al}$ MAS-NMR muestra como la evolución térmica de este tipo de compuestos origina la estructura espinela con cierto grado de inversión en función de la temperatura de tratamiento.

\section{AGRADECIMIENTOS}

Los autores desean expresar su agradecimiento al Servei Central d'Instrumentació Científica de la Universitat Jaume I por las facilidades prestadas en la caracterización de las muestras analizadas. Asimismo, al proyecto MAT-2000-1148C02-01 del MCyT por el apoyo económico prestado

\section{BIBLIOGRAFÍA}

1. F.Cavani, F.Trifiro, A.Vaccari "Hydrotalcite-type anionic clays: preparation, properties and applications" Catal.Today 11 pp.173-301 (1991).

2. A. de Roy, C.Forano, K.El-Malki, J.P.Besse, "Anionic Clays: Trends in Pillaring Chemistry" in Expanded Clays and Other Microporous Solids, ed. M.L.Ocelli and H.E.Robson, Van Nostrand Reinhold, New York pp.108-169 (1992).

3. V.Rives "Study of Layered Double Hydroxides by Thermal Methods" in Layered Double Hydroxides: Present and Future, ed. V.Rives, Nova Science Publisher Inc. New York pp.115-132 (2001).

4. S.Miyata "The synthesis of hydrotalcite-like compounds and their structures and physicochemical properties" Clays and Clay Minerals 23 pp.369-375 (1975).

5. M.del Arco, C.Martin, I.Martin, V.Rives, R.Trujillano "A FTIR spectroscopic study of surface acidity and basicity of mixed $\mathrm{Mg}$,Al-oxides obtained by thermal decomposition of hydrotalcite" Spectrochimica acta 49A(11) pp.1575-1582 (1992).

6. M.J.Hudson, S.Carlino, D.C. Apperley "Thermal conversion of a layered $(\mathrm{Mg} / \mathrm{Al})$ double hydroxide to the oxide" J.Mater.Chem. 5(2) pp.323-329 (1995).

7. F.M.Labajos, V.Rives "Thermal evolution of chromium(III) ions in hydrotalcite-like compounds" Inorg.Chem. 35 pp.5313-5318 (1996).

8. M.del Arco, R.Trujillano, V.Rives "Cobalt-iron hydroxicarbonates and their evolution to mixed oxides with spinel structure" J.Mater.Chem. 8(3) pp.761767 (1998).

9. E.Kanezaki "Thermal behaviour of the hydrotalcite-like layered structure of $\mathrm{Mg}$ and Al-layered double hydroxides with interlayer carbonate by means of in situ powder HTXRD and DTA/TG" Solid State Ionics 106 pp.279-284 (1998).

10. M.Jitianu, M.Balasoiu, R.Marchidan, M.Zaharescu, D.Crisan, M.Craiu "Thermal behaviour of hydrotalcite-like compounds: study of the resulting oxidic forms" Int. Journal of Inorg. Mater. 2 pp.287-300 (2000).

11. J.Rocha, M. del Arco, V.Rives, M.A.Ulibarri "Reconstruction of layered double hydroxides from calcined precursors: a Powder XRD and ${ }^{27} \mathrm{Al}-$ MASNMR study" J.Mater.Chem. 9 pp.2499-2503 (1999).

12. I.Nebot-Díaz "Estudio y caracterización de compuestos tipo espinela mediante rutas de síntesis no convencionales. Aplicación a la industria cerámica" Tesis doctoral, Universitat Jaume I, Castellón (España) 2001.

Recibido: 26.11 .01

Aceptado: 27.05.02

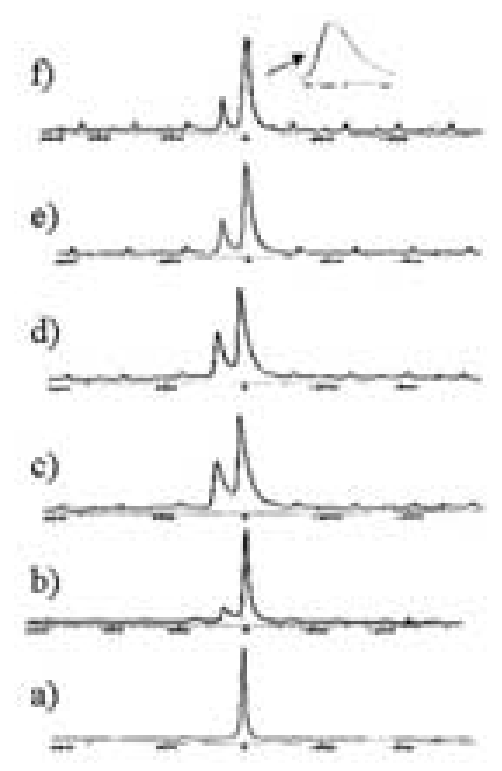

Figura 6. Espectros ${ }^{27} \mathrm{Al}-\mathrm{MASNMR}$ de a) muestra cruda, b) calcinada a $300^{\circ} \mathrm{C}$, c) $500^{\circ} \mathrm{C}$, d) $700^{\circ} \mathrm{C}$, e) $900^{\circ} \mathrm{C}$ y f) $1200^{\circ} \mathrm{C}$

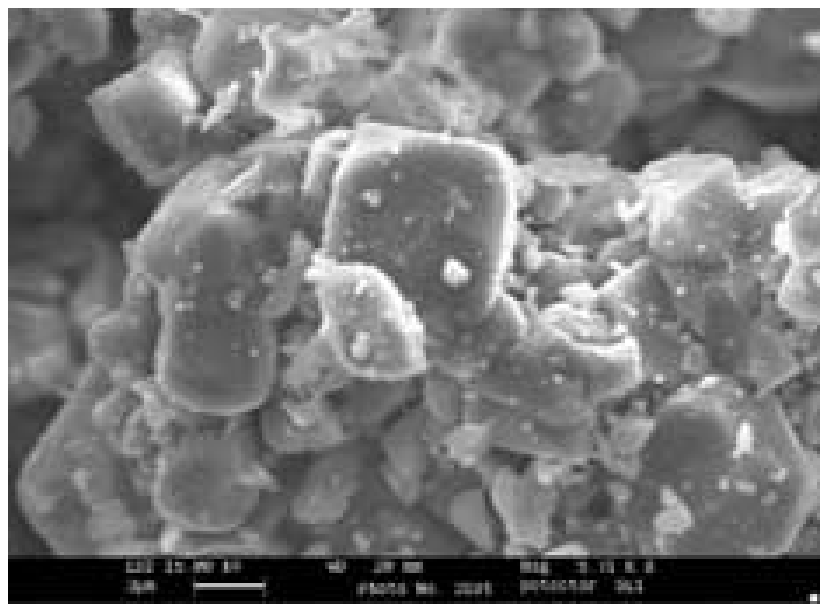

Figura 7. Micrografía MEB de la muestra Mg12 calcinada a $1200^{\circ} \mathrm{C}$

TABLA II. RELACIÓN DE INTENSIDADES ${ }^{27} \mathrm{Al}$ MAS-NMR DE LAS SEÑALES DE $\mathrm{Al}^{\mathrm{VI}} / \mathrm{Al}^{\mathrm{IV}}$ EN LA MUESTRA Mg12

\begin{tabular}{cc}
\hline $\mathbf{T}\left({ }^{\mathbf{0}} \mathbf{C}\right)$ & Relación $_{\mathbf{A l}}^{\mathbf{V I}} \mathbf{:} \mathbf{A l}^{\mathbf{I V}}$ \\
Cruda & $32: 1$ \\
300 & $4.2: 1$ \\
500 & $1.9: 1$ \\
700 & $2: 1$ \\
900 & $2: 1$ \\
1200 & $8.7: 1$ \\
\hline
\end{tabular}

Boletín de la Sociedad Española de Cerámica y Vidrio. Vol. 41 Núm. 4 Julio-Agosto 2002 